\title{
Refining Constraint Weighting
}

\author{
Hugues Wattez $^{* \dagger}$, Christophe Lecoutre* ${ }^{* \dagger}$, Anastasia Paparrizou*, Sébastien Tabary*† \\ ${ }^{*}$ CRIL, CNRS UMR 8188, Lens, France \\ ${ }^{\dagger}$ Université d'Artois, Lens, France \\ Email: \{wattez,lecoutre,paparrizou,tabary\}@ cril.fr
}

\begin{abstract}
Backtracking search is a complete approach that is traditionally used to solve instances modeled as constraint satisfaction problems. The space explored during search depends dramatically on the order that variables are instantiated. Considering that a perfect variable ordering might result to a backtrack-free search (i.e., finding backdoors, cycle cutsets), finding heuristics for variable ordering has always attracted research interest. For fifteen years, constraint weighting has been shown to be a successful approach for guiding backtrack search. In this paper, we show how the popular generic variable ordering heuristic dom/wdeg can be made more robust by taking finer information at each conflict: the "current" arity of the failing constraint as well as the size of the current domains of the variables involved in that constraint. Our experimental results show the practical interest of this refined variant of constraint weighting.
\end{abstract}

Index Terms-constraint satisfaction, search heuristics, constraint weighting

\section{INTRODUCTION}

Backtrack search remains a classical approach for solving instances of the Constraint Satisfaction Problem (CSP). It is based on a depth-first exploration, which is conducted by instantiating variables in sequence and backtracking when dead-ends occur. For efficiently exploring the search space, a property (called local consistency) is enforced at each step of the search so as to filter the domains of the variables; typically most of the constraints guarantees the property known as (generalized) arc consistency.

The order in which variables are chosen during the depthfirst traversal of the search space is decided by a variable ordering heuristic $H$. At each internal node of the search tree built by the backtrack search algorithm, the next variable $x$ is selected by $H$, and a value is assigned to $x$ according to a value ordering heuristic, which can simply be the lexicographic order over the domain of $x$. Choosing the right variable ordering heuristic for a given constraint network is a key issue in the design of constraint solvers, since different heuristics can lead to drastically different search trees.

For a long time, the most popular (variable ordering) heuristic was dom [8] that selects variables in sequence of increasing size of domain. However, fifteen years ago, modern adaptive heuristics were introduced: they take into account information collected along the part of the search space (tree) already explored. The two first proposed generic adaptive heuristics are impact [13] and wdeg [2]. The former relies on a measure of the effect of any assignment, and the latter associates a counter with each constraint (and indirectly, with each variable) indicating how many times any constraint led to a domain wipe-out. Counting-based heuristics [12] and activity-based search [10] are two more recent additional adaptive techniques for guiding search.

Currently, the constraint weighting variant dom/wdeg that additionally takes domain sizes into account, is considered as the most robust generic heuristic, as it is used by default in many constraint solvers (e.g., Choco). It certainly remains the state-of-the-art (as a generic heuristic) even if several attempts were made to further improve it. A first idea [5] was to learn weighting information during an initial phase in which variables are chosen at random and the search is repeatedly run to a fixed cutoff. This random probing method was intended to start the "real" search better informed after gathering information from different parts of the search space. Some other variants were also studied in [1]. By noting the constraint responsible of each value deletion (a kind of explanation), it is possible to implement different weighting strategies. For example, whenever there is a domain wipe-out on a variable $x$ while propagating constraint $c$, the weight of every constraint responsible for the removal of a value of $x$ is incremented. Another variant uses an aging mechanism, as in some SAT solvers, which periodically divides the value of all weights by a constant, thereby giving greater importance to conflicts discovered recently. Surprisingly, the "basic" dom/wdeg heuristic remained very competitive compared to such attractive variants.

A specific variant of constraint weighting was shown to be successful for job-shop scheduling problems [6]: by reasoning from the domain sizes associated with the variables denoting the starting times of tasks, the proposed weightingbased heuristic was shown to be better informed and to yield particularly strong performance for scheduling. Because it was observed that the efficiency of dom/wdeg may deteriorate when problem instances contain many constraints of large arity (because it loses its ability to discriminate variables), a possible approach [9] is to weight a conflict set rather than the entire scope of a failed constraint. Although this approach is stimulating, it is unfortunately not generic since one has to conceive a specific procedure for each type of (global) constraints.

More recently, a new competitive heuristic [7], called CHS, has been proposed by exploiting the history of search failures. Techniques coming from reinforcement learning are used to 
make an exponential recency weighted average in order to estimate the evolution of the hardness of constraints throughout the search. In brief, this heuristic gives a higher reward to constraints that fail regularly over short periods.

The paper is organized as follows. After some preliminaries, we introduce classical variable ordering heuristics. Next, we show how to refine constraint weighting, and demonstrate the practical interest of our approach. Finally, we conclude.

\section{PRELIMINARIES}

A constraint network $P$ is composed of a finite set of variables $\mathcal{X}$, and a finite set of constraints $\mathcal{C}$. Each variable $x$ must be assigned a value from its current domain, denoted by $\operatorname{dom}(x)$; the initial domain of $x$ is denoted by $\operatorname{dom}^{\text {init }}(x)$. Each constraint $c$ represents a mathematical relation over an ordered set of variables, called the scope of $c$, and denoted by $\operatorname{scp}(c)$. The arity of a constraint $c$ is the size of its scope. The degree of a variable $x$ is the number of constraints of $\mathcal{C}$ involving $x$.

A solution to $P$ is the assignment of a value to each variable of $\mathcal{X}$ such that all constraints of $\mathcal{C}$ are satisfied. A constraint network is satisfiable iff it admits at least one solution. The Constraint Satisfaction Problem (CSP) is to determine whether a given constraint network is satisfiable, or not. A classical approach for solving this NP-complete problem is to perform a depth-first search with backtracking, while enforcing a property called (generalized) arc consistency [11] after each taken decision. This procedure, called Maintaining Arc Consistency (MAC) [14], builds a binary search tree $\mathcal{T}$ : for each internal node $\nu$ of $\mathcal{T}$, a pair $(x, v)$ is selected where $x$ is a variable and $v$ is a value in $\operatorname{dom}(x)$. Then, two cases are considered: the assignment $x=v$ (positive decision) and the refutation $x \neq v$ (negative decision). In this paper, we shall be interested in the future variables of a constraint $c$, denoted by $\operatorname{fut}(c)$, which are the variables at the current node of the search tree that have not been explicitly assigned by MAC.

Backtrack search algorithms that rely on deterministic variable ordering heuristics have been shown to exhibit heavytailed behavior on both random and structured CSP instances [4]. This issue can be alleviated using randomization and restart strategies, which respectively incorporate some random choices in the search process, and iteratively restart the computation from the beginning, with a different variable ordering.

\section{VARIABle ORdering Heuristics}

We provide in this section a quick overview of popular general-purpose search heuristics. The simple variable ordering heuristic dom [8], which selects variables in sequence of increasing size of domain, has long been considered as the most robust backtrack search heuristic. However, fifteen years ago, modern adaptive heuristics were introduced: they take into account information collected along the part of the search space (tree) already explored.

In this paper, we shall mainly focus our attention to the very popular heuristic wdeg, and its variant dom/wdeg. As a baseline, we shall also consider impact and activity, which are defined as follows:

- impact, or IBS (Impact-Based Search) selects in priority the variable with the highest impact. The impact of a variable $x$ gives a measure about the importance of $x$ in reducing the search space [13]. The size of the search space of $P$ is the product of all current domain sizes:

$$
\operatorname{size}(P)=\prod_{x \in \mathcal{X}}|\operatorname{dom}(x)|
$$

The impact $I$ of a variable assignment $x=a$ on $P$ is computed as follows:

$$
I(x=a)=1-\frac{\operatorname{size}\left(P^{\prime}\right)}{\operatorname{size}(P)}
$$

where $P^{\prime}=A C\left(\left.P\right|_{x=a}\right)$ denotes the $\mathrm{CN}$ obtained after assigning $x$ to $a$ and enforcing (generalized) arc consistency. Note that if $P^{\prime}$ leads to a failure, then $I(x=a)=1$. It is easy to see that this heuristic can be used for value selection as well.

- activity, or ABS (Activity-Based Search) selects in priority the variable with the highest activity. The activity of a variable $x$ is roughly measured by the number of times the domain of $x$ is reduced during search [10]. This heuristic is motivated by the key role of propagation in constraint programming and relies on a decaying sum to forget the oldest statistics progressively. The activities are initialized by making random probing in the search space.

- CHS (Conflict-History Search), selects in priority variables appearing in recent failures. All failures are registered with a timestamp. More precisely, CHS maintains for each constraint $c$, a score $q(c)$ and updates it at every domain wipeout with an exponential recency weighted average:

$$
q(c)=(1-\alpha) \times q(c)+\alpha \times r(c)
$$

where $\alpha=0.4$ (decreasing as time goes by) and $r(c)$ is the reward gives when a domain wipeout occurred. Reward is higher when the constraint entered frequently in conflict :

$$
r(c)=\frac{1}{\# \operatorname{Conflicts}-\operatorname{Conflict}(\mathrm{c})+1}
$$

\#Conflicts is the total number of conflicts and Conflict(c) stores the last \#Conflicts value where $c$ led to a failure. The conflict history score (chv) of a variable $x$ which will be used in selecting the branching variable is given by:

$$
\operatorname{chv}(x)=\frac{\sum_{c \in \mathcal{C}: x \in \operatorname{scp}(c) \wedge \mid \text { fut }(c) \mid>1} q(c)+\delta}{|\operatorname{dom}(x)|}
$$

where $\delta$ is a positive real number close to 0 that avoid random selection at the beginning of search. Thus, the branching will be oriented according to the degree of the variables.

To introduce wdeg and dom/wdeg, we need to describe the way constraint propagation is run each time a decision is taken by the backtrack search algorithm. Algorithm 1 describes 


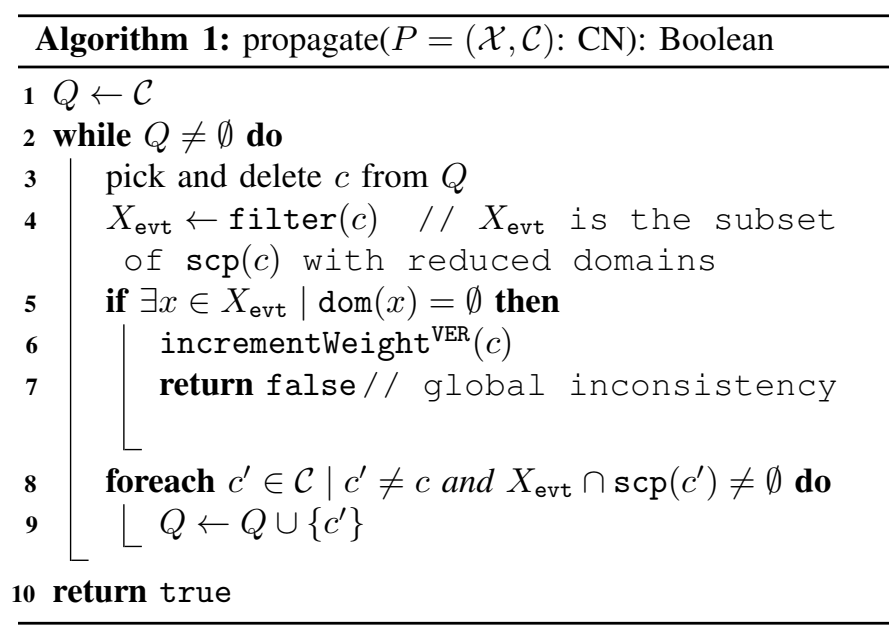

a basic propagation scheme based on the use of a queue of constraints. Other schemes exists in the literature, but this is not an important issue for introducing constraint weighting. This algorithm is then applied at the beginning of the search and systematically each time a decision is taken. Initially the queue $Q$ contains the whole set of constraints of the constraint network. Then, each constraint $c$ in $Q$ is picked in turn and a filtering process is applied from $c$ : typically, this is for enforcing arc-consistency by calling Function filter(c) at Line 4 . The call to this function returns a subset of variables of the scope of $c$, denoted by $X_{\text {evt }}$, whose domains have been modified (i.e., such that at least one value has been removed from these domains). By means of $X_{\text {evt }}$, we can update $Q$ so as to ensure constraint propagation is run until a fixed point is reached. If ever the domain of one variable of $X_{\text {evt }}$ becomes empty, it simply means that a conflict occurred (a dead-end has been identified) and so, a backtrack is required. This is triggered by the returned Boolean value false, after having called the function incrementWeight ${ }^{\mathrm{VER}}$ with the culprit constraint (responsible of the domain wipeout) passed as a parameter. In the initial paper [2], the principle of constraint weighing is very simple: the weight of the culprit constraint $c$, denoted by $c$.weight, is incremented by 1 , as shown in Algorithm 2 (here, VER written as a superscript at Line 6 of Algorithm 1 corresponds to 2004). To summarize, each constraint $c$ admits a weight, initially set to 1 , which is incremented whenever a domain wipeout occurs while filtering c.

\begin{tabular}{l}
\hline Algorithm 2: incrementWeight ${ }^{2004}(c:$ Constraint $)$ \\
\hline 1 c.weight $\leftarrow c$.weight +1
\end{tabular}

\begin{tabular}{l}
\hline Algorithm 3: incrementWeight ${ }^{\mathrm{AbsCon}}(c:$ Constraint $)$ \\
\hline 1 foreach $x \in \operatorname{fut}(c)$ do \\
$2 \quad$ c.weights $[\mathrm{x}] \leftarrow c$.weights $[\mathrm{x}]+1$ \\
\hline
\end{tabular}

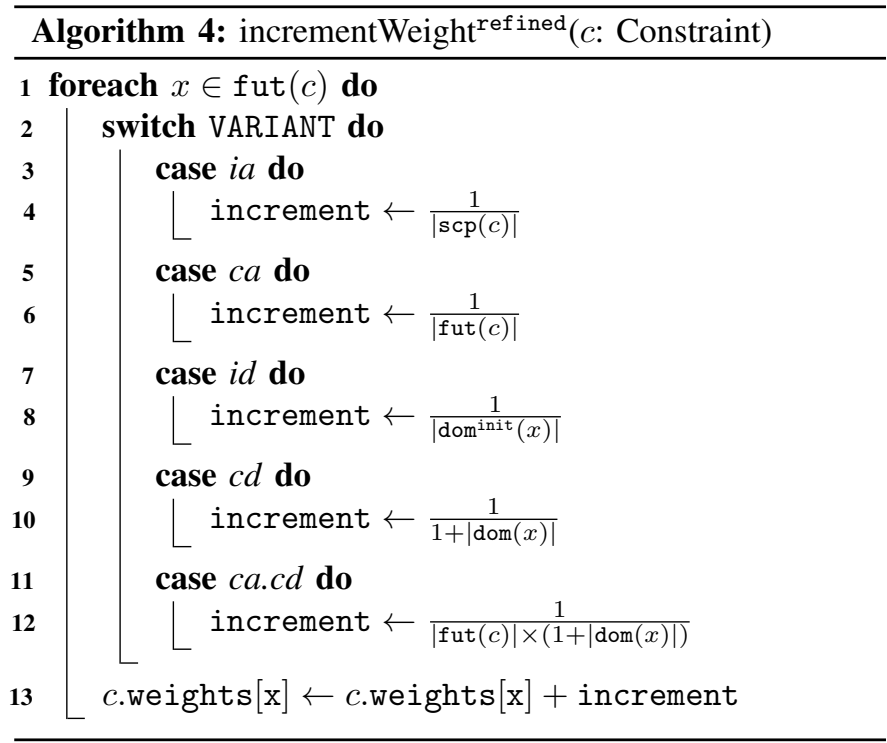

The heuristics wdeg and dom/wdeg are defined as follows: - wdeg selects in priority the future variable with the highest 'weighted degree'. Each variable $x$ is given a weighted degree, which is the sum of the weights over all constraints involving $x$ and at least another future variable. For each future variable $x$, the score of $x$ according to wdeg is:

$$
\sum_{c \in \mathcal{C}: x \in \operatorname{scp}(c) \wedge \mid \text { fut }(c) \mid>1} c . \text { weight }
$$

- dom/wdeg selects in priority the future variable with the smallest ratio 'current domain size to weighted degree'. For each future variable $x$, the score of $x$ according to $\mathrm{dom} /$ wdeg is:

$$
\frac{|\operatorname{dom}(x)|}{\sum_{c \in \mathcal{C}: x \in \operatorname{scp}(c) \wedge \mid \text { fut }(c) \mid>1} c . \text { weight }}
$$

To break ties, which correspond to sets of variables that are considered as equivalent by the heuristic, one can use a second criterion (e.g., the dynamic degree of each variable). However, for adaptive heuristics, it is usual to use lexico, meaning that the first encountered variable with the best score is selected.

\section{REFINING WEIGHTED DEGREES}

The heuristic dom/wdeg is very simple to be implemented while being quite robust. However, this is not exactly the version that is implemented in the constraint solver $\mathrm{AbsCon}$. Indeed, it was observed experimentally that it was more effective to consider only the future variables involved in a culprit constraint. Technically, instead of associating a global weight $c$.weight with each constraint $c$, one can introduce a local weight $c$.weight $[x]$ to be associated with each variable $x$ in $\operatorname{scp}(c)$. Hence, when a conflict occurs, instead of incrementing the weight $c$.weight of the culprit constraint, one can decide to increment the local weight $c$.weight $[x]$ of each future variable involved in $\operatorname{scp}(c)$. Because each variable has now its specific weight in each constraint, the score of a future variable $x$ becomes: 


$$
\sum_{c \in \mathcal{C}: x \in \operatorname{scp}(c) \wedge \mid \text { fut }(c) \mid>1} c . \text { weight }[\mathrm{x}]
$$

for wdeg and:

$$
\frac{|\operatorname{dom}(x)|}{\sum_{c \in \mathcal{C}: x \in \operatorname{scp}(c) \wedge|\operatorname{fut}(c)|>1} c . \text { weight }[\mathrm{x}]}
$$

for dom/wdeg.

Constraint weighting is now given by Algorithm 3 that describes the function called at Line 6 of Algorithm 1. To distinguish between the 2004 version and the AbsCon version, we shall refer to the heuristics of the 2004 initial paper with wdeg $^{2004}$ and dom/wdeg ${ }^{2004}$.

Even if dom/wdeg slightly outperforms dom/wdeg ${ }^{2004}$ (this is shown in Section V), one may regret that constraint weighting remains very simplistic and does not differentiate between constraints. For instance, characteristics like the arity of the constraints and the state of the domains of the participant variables are totally ignored as the increment is static (i.e., 1). This is why we propose to refine constraint weighting by exploiting such information. More specifically, we introduce four variants in Algorithm 4 as follows:

- ia is the variant in which the 'initial' arity of the constraints is used.

- ca is the variant in which the 'current' arity (i.e., the number of future variables) of the constraints is used.

- id is the variant in which the (size of the) initial domains of the future variables is used.

- $\mathrm{cd}$ is the variant in which the (size of the) current domains of the future variables is used.

- ca. cd combines both current arity and current domains.

These different variants are described by Algorithm 4 .

\section{EXPERIMENTAL RESULTS}

We have conducted a first experiment with all available CSP series (82) from the XCSP3 [3] archive (http://xcsp.org), which contains 9, 243 CSP instances (referred to as ALL). We have also conducted two additional experiments by considering the instances from the main CSP track at the 2017 XCSP3 competition (COMP-17 composed of aim, AllInterval, bdd, Bibd, Blackhole, bmc, bqwh, Cabinet, CarSequencing, ColouredQueens, composed, CostasArray, CoveringArray, Crossword, CryptoPuzzle, DeBruijnSequence, DiamondFree, Domino, driverlogw, Dubois, ehi, Fischer, geometric, gp10, GracefulGraph, Hanoi, Haystacks, jnh, Kakuro, Knights, KnightTour, Langford, LangfordBin, lard, MagicHexagon, MagicSequence, MagicSquare, MarketSplit, mdd, MultiKnapsack, Nonogram, NumberPartitioning, Ortholatin, Pb, pigeonsPlus, Primes, PropStress, QuasiGroup, QueenAttacking, Queens, QueensKnights, qwh, RadarSurveillance, rand, RectPacking, reg, Renault, RenaultMod, Rlfap, RoomMate, Sadeh, Sat, SchurrLemma, SocialGolfers, SportsScheduling, Steiner3, StripPacking, Subisomorphism, Sudoku, SuperQueens, SuperSadeh, SuperTaillard, TravellingSalesman, Wwtpp) and 2018 XCSP3 competition (COMP18 composed of Bibd, CarSequencing, ColouredQueens, Crossword, Dubois, Eternity, frb, GracefulGraph, Haystacks,
LangfordBin, MagicHexagon, MisteryShopper, Pb, QuasiGroup, Rlfap, SocialGolfers, SportsScheduling, StripPacking, Subisomorphism). These instances have been launched on a cluster equipped with $2.66 \mathrm{GHz}$ Intel Xeon and $32 \mathrm{~GB}$ RAM nodes. The constraint solver used for our experiments is AbsCon where our new constraint weighting variants and $\mathrm{CHS}$ have been implemented. The timeout was set to 20 minutes.

TABLE I

COMPARISON OF HEURISTICS IN TERMS OF NUMBER OF SOLVED INSTANCES (\#INST.) AND SEVERAL TIME METRICS [COMP-18]

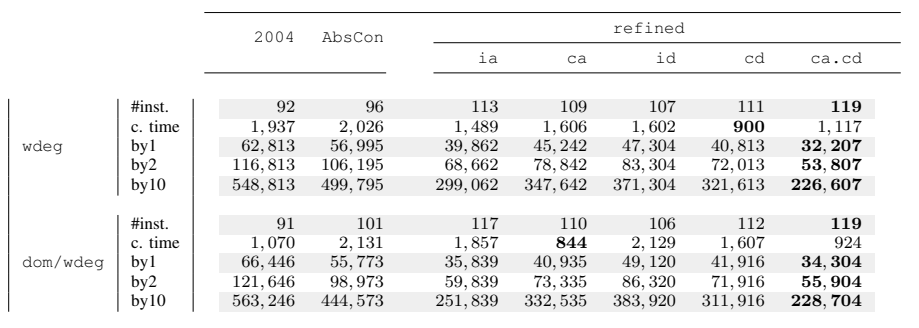

In Table I, the new constraint weighting variants proposed in that paper are compared with the classical wdeg and dom/wdeg heuristics (2004 and AbsCon versions). The comparison is given by the number of solved instances (within 1,200 seconds) as well as by several time metrics: the cumulated CPU time (c. time) computed from instances solved by all methods, and the cumulated CPU times (by1, by2, by10) computed from all instance by considering for unsolved instances a 'solving' time equal to $x \times 1,200$ for $x=1$, $x=2$ and $x=10$, respectively. Numbers given in bold face correspond to the best obtained results. In Table I, we can observe that classical heuristics are outperformed by the new variants. Notably, the variant ca.cd is clearly the best one as it allows us to solve $18 \%$ more instances than the best classical heuristic dom/wdeg ${ }^{\text {AbsCon }}$ (119 vs 101). Such results are confirmed by Table II on the CSP 2017 competition instances.

TABLE II

COMPARISON OF HEURISTICS IN TERMS OF NUMBER OF SOLVED INSTANCES (\#INST.) AND SEVERAL TIME METRICS [COMP-17]

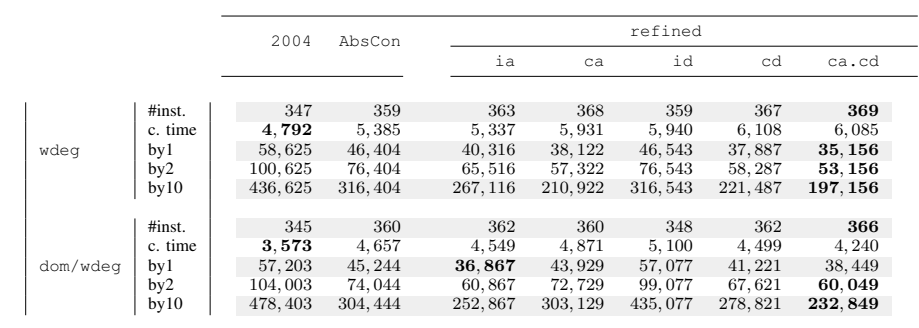

In Table III, we provide some details about specific series. Due to lack of space, we decided to only keep c. time as time metric because we find it to be the most relevant one. For the lack of clarity, some series have been discarded from this table because we obtained rather similar results whatever the heuristic is used. However, note that these series are taken into account when displaying the total number of solved instances 
TABLE III

COMPARISON OF HEURISTICS IN TERMS OF NUMBER OF SOLVED INSTANCES (\#INST.) AND CUMULATED CPU TIME (C. TIME) [ALL]

\begin{tabular}{|c|c|c|c|c|c|c|c|c|c|c|}
\hline & \multicolumn{2}{|c|}{ wdeg 2004} & \multicolumn{2}{|c|}{ wdeg Abscon } & \multicolumn{2}{|c|}{ dom/wdeg 2004} & \multicolumn{2}{|c|}{ dom/wdeg Abscon } & \multicolumn{2}{|c|}{ wdeg ${ }^{\mathrm{ca}} \cdot \mathrm{cd}$} \\
\hline & \#inst & c. time & \#inst & c. time & \#inst & c. time & \#inst & c. time & \#inst & c. time \\
\hline AllInterval & 5 & 1,094 & 14 & 397 & 15 & 20 & 15 & 845 & 15 & 20 \\
\hline bdd & 48 & 584 & 48 & 926 & 48 & 583 & 48 & 1,028 & 48 & 952 \\
\hline Bibd & 30 & 1,827 & 82 & 623 & 27 & 1,019 & 88 & 967 & 84 & 209 \\
\hline Blackhole & 1 & 0 & 20 & 0 & 0 & 0 & 14 & 0 & 20 & 0 \\
\hline bmc & 12 & 3,716 & 16 & 195 & 12 & 4,022 & 16 & 250 & 16 & 180 \\
\hline Cabinet & 20 & 131 & 20 & 138 & 20 & 177 & 20 & 283 & 20 & 115 \\
\hline CarSequencing & 31 & 248 & 37 & 383 & 19 & 872 & 30 & 565 & 48 & 446 \\
\hline ColouredQueens & 0 & 0 & 1 & 0 & 0 & 0 & 0 & 0 & 1 & 0 \\
\hline CostasArray & 3 & 374 & 5 & 89 & 4 & 186 & 4 & 825 & 4 & 169 \\
\hline CoveringArray & 1 & 207 & 2 & 73 & 4 & 4 & 4 & 3 & 3 & 3 \\
\hline Crossword & 169 & 11,852 & 157 & 18,902 & 190 & 3,631 & 185 & 4,260 & 176 & 11,628 \\
\hline DeBruijnSequence & 5 & 516 & 6 & 495 & 6 & 358 & 6 & 396 & 5 & 805 \\
\hline DiamondFree & 17 & 2,205 & 22 & 349 & 18 & 3,242 & 22 & 493 & 22 & 208 \\
\hline DistinctVectors & 3 & 891 & 3 & 32 & 3 & 1,057 & 3 & 96 & 3 & 34 \\
\hline Dubois & 8 & 81 & 19 & 36 & 8 & 120 & 6 & 435 & 20 & 60 \\
\hline frb & 19 & 2,417 & 20 & 2,355 & 28 & 2,672 & 29 & 2,661 & 19 & 2,547 \\
\hline GracefulGraph & 4 & 281 & 7 & 59 & 8 & 204 & 7 & 50 & 8 & 28 \\
\hline Knights & 7 & 438 & 7 & 520 & 7 & 309 & 7 & 426 & 5 & 683 \\
\hline KnightTour & 17 & 20 & 16 & 25 & 4 & 1,299 & 10 & 37 & 18 & 16 \\
\hline Langford & 11 & 950 & 12 & 704 & 11 & 1,082 & 12 & 1,035 & 12 & 898 \\
\hline LangfordBin & 1 & 6 & 1 & 36 & 1 & 5 & 1 & 13 & 10 & 3 \\
\hline MagicHexagon & 3 & 89 & 9 & 67 & 9 & 7 & 18 & 10 & 16 & 7 \\
\hline MagicSequence & 14 & 819 & 14 & 2,542 & 14 & 1,780 & 14 & 1,660 & 14 & 2,137 \\
\hline MagicSquare & 11 & 626 & 21 & 407 & 32 & 163 & 43 & 58 & 41 & 150 \\
\hline MarketSplit & 10 & 396 & 7 & 1,212 & 10 & 449 & 9 & 620 & 9 & 624 \\
\hline mdd & 33 & 3,476 & 29 & 4,922 & 32 & 3,298 & 27 & 3,604 & 29 & 4,984 \\
\hline MultiKnapsack & 11 & 64 & 9 & 416 & 11 & 52 & 9 & 1,066 & 10 & 86 \\
\hline NumberPartitioning & 38 & 258 & 38 & 588 & 15 & 494 & 29 & 3,926 & 38 & 501 \\
\hline Ortholatin & 4 & 28 & 4 & 16 & 2 & 5 & 2 & 8 & 3 & 29 \\
\hline $\mathrm{Pb}$ & 3 & 299 & 4 & 705 & 3 & 117 & 4 & 105 & 6 & 146 \\
\hline pigeonsPlus & 13 & 3,012 & 14 & 2,178 & 15 & 1,583 & 15 & 1,466 & 15 & 1,489 \\
\hline Primes & 8 & 60 & 15 & 80 & 13 & 209 & 18 & 94 & 16 & 239 \\
\hline qcp & 11 & 808 & 11 & 334 & 11 & 622 & 13 & 480 & 13 & 578 \\
\hline QuasiGroup & 4 & 327 & 4 & 476 & 5 & 125 & 6 & 210 & 5 & 248 \\
\hline QueensKnights & 9 & 147 & 9 & 150 & 5 & 500 & 6 & 387 & 8 & 206 \\
\hline qwh & 43 & 8,303 & 43 & 4,291 & 51 & 3,487 & 47 & 5,178 & 52 & 3,876 \\
\hline RadarSurveillance & 40 & 1,798 & 40 & 1,688 & 40 & 2,483 & 40 & 2,348 & 41 & 1,922 \\
\hline Rlfap & 6 & 652 & 7 & 533 & 6 & 518 & 7 & 397 & 7 & 584 \\
\hline RoomMate & 13 & 5,457 & 14 & 5,239 & 11 & 5,740 & 14 & 5,577 & 14 & 5,167 \\
\hline SocialGolfers & 59 & 1,744 & 56 & 2,105 & 44 & 3,916 & 51 & 1,299 & 61 & 1,207 \\
\hline StripPacking & 2 & 0 & 5 & 0 & 0 & 0 & 3 & 0 & 7 & 0 \\
\hline Subisomorphism & 206 & 2,241 & 162 & 18,193 & 204 & 4,616 & 200 & 2,380 & 211 & 2,099 \\
\hline SuperQueens & 1 & 657 & 1 & 739 & 1 & 292 & 1 & 282 & 1 & 892 \\
\hline SuperSadeh & 10 & 163 & 11 & 245 & 9 & 225 & 9 & 612 & 9 & 182 \\
\hline SuperTaillard & 39 & 2,258 & 37 & 1,960 & 41 & 3,847 & 37 & 2,570 & 37 & 2,386 \\
\hline TravellingSalesman & 18 & 988 & 18 & 1,157 & 18 & 281 & 18 & 339 & 18 & 1,262 \\
\hline Wwtpp & 229 & 5,562 & 244 & 2,119 & 243 & 9,380 & 242 & 15,269 & 240 & 2,049 \\
\hline Total & 1,465 & 75,375 & 1,559 & 84,436 & 1,486 & 74,116 & 1,617 & 72,547 & 1,694 & 60,482 \\
\hline
\end{tabular}

(last line of the table). For the remaining series, we also discarded 'easy' instances, which are CSP instances solved by all heuristics by less than 10 seconds. In each row, the highest number of solved instances is written in bold, except when all heuristics solve the same instances, in which case the c.time is given in bold. Once again, we can observe that wdeg ${ }^{\text {ca.cd }}$ is the best variant.

Figure 1 shows a scatterplot allowing us to compare the overall respective behavior of dom/wdeg ${ }^{\mathrm{AbsC}} \mathrm{Con}$ and wdeg $^{\text {ca.cd }}$. For our comparison, we used the set COMP$17+18$ containing instances coming from both the 2017 and 2018 XCSP instances (main CSP track). Each dot in this figure represents a CSP instance. The coordinates of this dot are defined by: on the horizontal axis, the CPU time (in seconds) required to solve the instance with $\mathrm{dom} / \mathrm{wdeg}^{\mathrm{AbsCon}}$ and on the vertical axis, the CPU time required to solve the instance with wdeg ${ }^{\text {ca.cd }}$. One can observe that more instances are located at the bottom-right side of the figure, meaning that wdeg $^{\mathrm{ca} . c d}$ is usually more efficient. Also, note the presence of many dots along the right border, indicating that these instances have not been solved (within 1,200 seconds) by the classical heuristic dom/wdeg ${ }^{\mathrm{AbsC}}$. The same trend can be observed in Figure 2, when comparing CHS and wdeg ${ }^{\mathrm{ca} . c d}$, even if results are closer. When comparing these two heuristics 


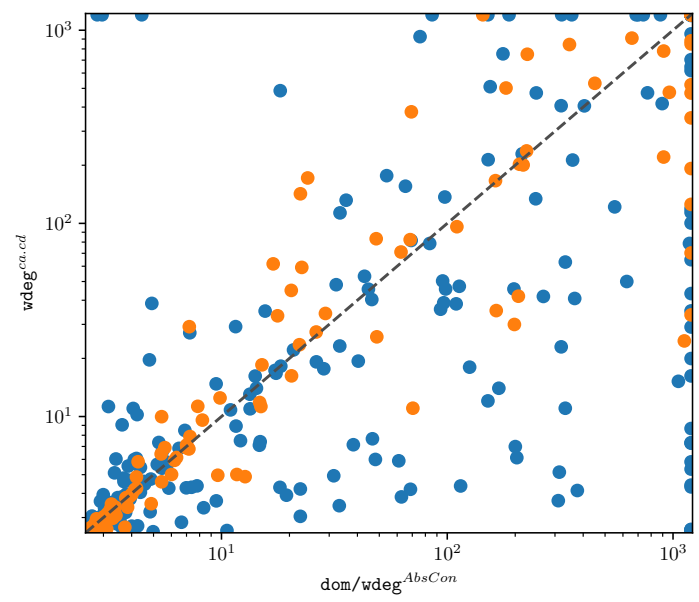

Fig. 1. Comparing dom/wdeg ${ }^{\text {Abscon }}$ and wdeg ${ }^{\text {ca.cd }}[$ COMP-17+18]

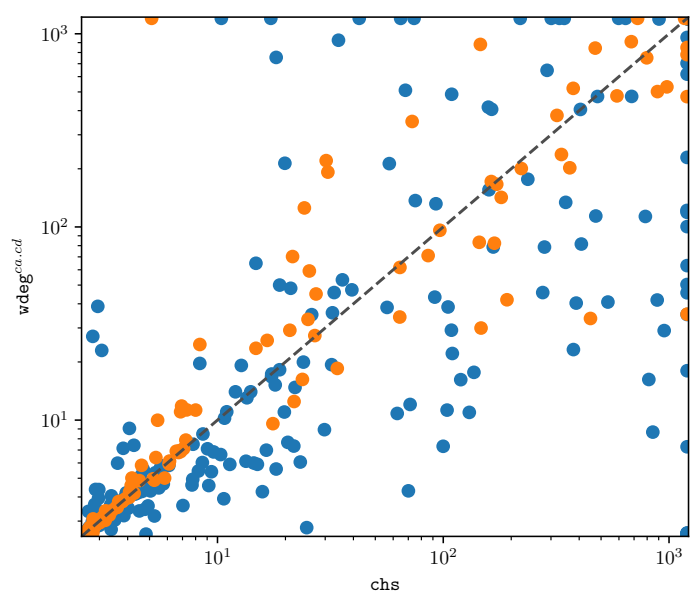

Fig. 2. Comparing CHS and wdeg ${ }^{\mathrm{ca} . c d}[\mathrm{COMP}-17+18]$

on the overall set of instances (ALL), the c. time of wdeg ${ }^{\text {ca.cd }}$ is decreased by $32 \%$ and 59 additional instances are solved.

The cactus plot in Figure 3 shows the performance of all popular generic heuristics and wdeg ${ }^{\mathrm{ca} . c d}$ on COMP-17+18. It displays the number of solved instances (on the horizontal axis) per unit of time (on the vertical axis). On the left of the figure, we can find the least effective heuristics, namely, impact, activity, wdeg ${ }^{2004}$ and $\mathrm{dom} / \mathrm{wdeg}^{2004}$ that behave rather similarly. In the middle of the figure, we have wdeg ${ }^{\mathrm{AbsCon}}$ and dom/wdeg ${ }^{\mathrm{AbsCon}}$, as implemented (and used by default) in AbsCon. Finally, CHS and wdeg ${ }^{\text {ca.cd }}$ are clearly the most efficient heuristics since they are situated on the right.

Figure 4 focuses on constraint weighting variants (comparing very classical heuristics with our new best variant $\mathrm{ca} \cdot \mathrm{cd}$ ). Clearly, wdeg ${ }^{c a . c d}$ appears to be the most robust heuristic based on constraint weighting.

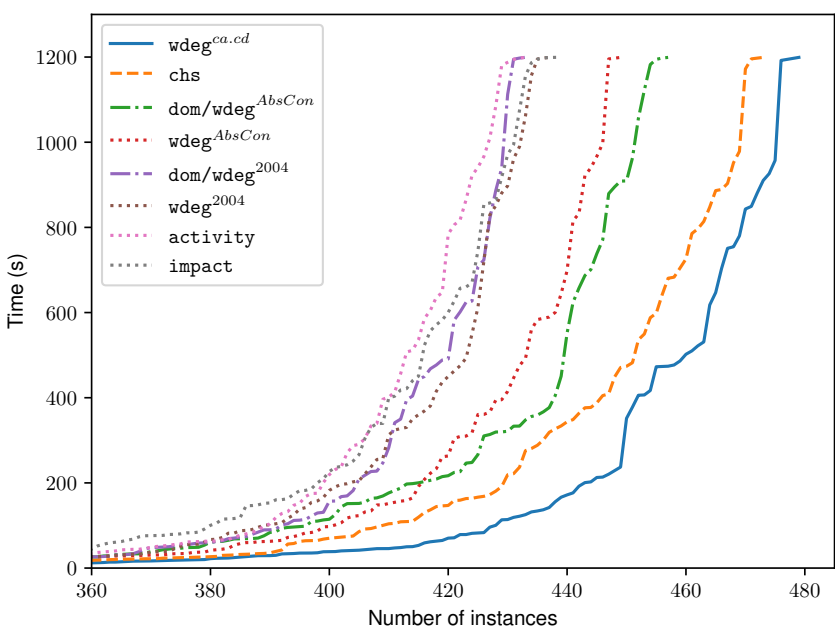

Fig. 3. Comparing popular heuristics and wdeg ${ }^{\text {ca.cd }}[$ COMP-17+18]

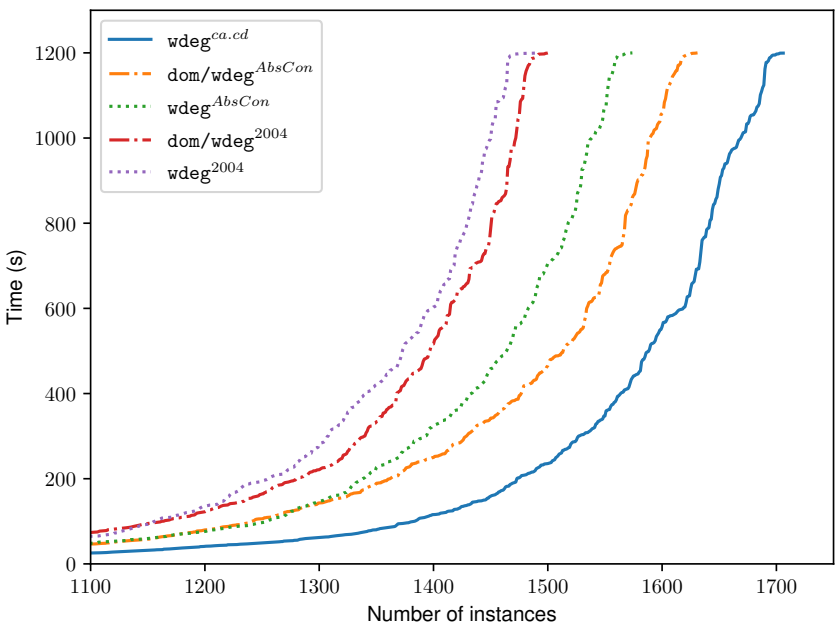

Fig. 4. Comparing popular heuristics and wdeg ${ }^{\mathrm{ca} . c d}$ [ALL]

\section{CONCLUSION}

In this paper, we have revisited constraint weighting that is known to be a robust generic approach to guide backtrack search. By refining the way weights of constraints (and variables) are updated by taking into account both constraint arities and sizes of variable domains, we show how the popular heuristic dom/wdeg can be improved. We think that $\mathrm{dom} / \mathrm{wdeg}^{\text {ca.cd }}$ is the most robust generic (variable ordering) heuristic to be used for solving instances of constraint satisfaction problems.

\section{ACKNOWLEDGMENT}

The authors are grateful to the anonymous reviewers for their comments. This work has been supported by the project CPER Data from the region "Hauts-de-France".

\section{REFERENCES}

[1] T. Balafoutis and K. Stergiou. On conflict-driven variable ordering heuristics. In Proceedings of CSCLP'08, 2008. 
[2] F. Boussemart, F. Hemery, C. Lecoutre, and L. Sais. Boosting systematic search by weighting constraints. In Proceedings of ECAI'04, pages 146150,2004

[3] F. Boussemart, C. Lecoutre, and G. Audemard C. Piette. XCSP3: an integrated format for benchmarking combinatorial constrained problems. CoRR, abs/1611.03398, 2016.

[4] C. Gomes, B. Selman, N. Crato, and H. Kautz. Heavy-tailed phenomena in satisfiability and constraint satisfaction problems. Journal of Automated Reasoning, 24:67-100, 2000.

[5] D. Grimes and R.J. Wallace. Learning to identify global bottlenecks in constraint satisfaction search. In Proceedings of FLAIRS'07, 2007.

[6] Diarmuid Grimes and Emmanuel Hebrard. Solving variants of the job shop scheduling problem through conflict-directed search. INFORMS Journal on Computing, 27:268-284, 042015.

[7] Djamal Habet and Cyril Terrioux. Conflict history based search for constraint satisfaction problem. In Proceedings of the 34th ACM/SIGAPP Symposium on Applied Computing, SAC '19, pages 1117-1122, New York, NY, USA, 2019. ACM.

[8] R. Haralick and G. Elliott. Increasing tree search efficiency for constraint satisfaction problems. Artificial Intelligence, 14:263-313, 1980.

[9] Emmanuel Hebrard and Mohamed Siala. Explanation-based weighted degree. pages 167-175, 052017.

[10] L. Michel and P. Van Hentenryck. Activity-based search for black-box constraint programming solvers. In Proceedings of CPAIOR'12, pages 228-243, 2012.

[11] Ugo Montanari. Network of constraints : Fundamental properties and applications to picture processing. Information Science, 7:95-132, 1974

[12] G. Pesant, C.-G. Quimper, and A. Zanarini. Counting-based search: Branching heuristics for constraint satisfaction problems. Journal of Artificial Intelligence Research, 43:173-210, 2012.

[13] P. Refalo. Impact-based search strategies for constraint programming. In Proceedings of $C P^{\prime} 04$, pages 557-571, 2004.

[14] D. Sabin and E.C. Freuder. Contradicting conventional wisdom in constraint satisfaction. In Proceedings of CP'94, pages 10-20, 1994. 